\title{
Argumentation et rationalité dans l'étude de textes talmudiques : les interactions dyadiques dans les Yeshivas
}

Argumentation and Rationality in the Study of Talmudic Texts

\section{Baruch Schwarz}

\section{(2) OpenEdition Journals}

Electronic version

URL: https://journals.openedition.org/aad/4647

DOI: $10.4000 /$ aad. 4647

ISSN: 1565-8961

\section{Publisher}

Université de Tel-Aviv

\section{Electronic reference}

Baruch Schwarz, "Argumentation et rationalité dans l'étude de textes talmudiques : les interactions dyadiques dans les Yeshivas", Argumentation et Analyse du Discours [Online], 25 | 2020, Online since 15 October 2020, connection on 07 December 2022. URL: http://journals.openedition.org/aad/4647 ; DOI: https://doi.org/10.4000/aad.4647

This text was automatically generated on 17 October 2020

\section{(†)

Creative Commons - Attribution-NonCommercial-NoDerivatives 4.0 International - CC BY-NC-ND 4.0 https://creativecommons.org/licenses/by-nc-nd/4.0/ 


\title{
Argumentation et rationalité dans l'étude de textes talmudiques : les interactions dyadiques dans les Yeshivas
}

\author{
Argumentation and Rationality in the Study of Talmudic Texts
}

\section{Baruch Schwarz}

\section{Introduction}

1 Dans la culture juive traditionnelle, l'étude et l'interprétation des textes sacrés ne sont pas simplement une pratique, il s'agit d'une mitzwah - une obligation essentielle. Cette obligation a une révélation pour origine : la tradition raconte les quarante jours et quarante nuits pendant lesquels Moïse se plongea dans les nuées du mont Sinaï pour apprendre de son Créateur le sens des commandements qu'Il venait de promulguer pour son peuple. Après cette période, Moïse descendit de la montagne pour transmettre les secrets que lui avait révélés son étude.

Cette transmission n'a pas simplement trait à un contenu, mais aussi à une forme : la connaissance émerge d'un moment dialogique; l'élève entend le maître - la source, mais cette source ne se tarit pas ; au contraire, elle peut même faire émerger, dans le dialogue que le maître et l'élève perpétuent hic et nunc, des significations qui vont audelà de ce que le maître avait entendu et compris lorsqu'il était lui-même élève. Le dialogue est donc une technique d'enrichissement infini. Il y a là un angle d'attaque propre à la rationalité pratique, l'établissement d'un rapport entre la temporalité et le dynamisme de l'argumentation.

L'exil qui suit la destruction du Temple transpose le centre de gravité de la tradition en Babylonie. La chaîne de dialogues entre maître et élève s'en trouve rompue. Selon Fischer (2009), le caractère hautement dialectique du Talmud (de Babylone) exprime la 
conscience qu'ont les Sages de cette époque que ce qui a été directement entendu de la tradition est perdu à tout jamais, et que le seul moyen de s'en approcher est de discuter des rapports écrits de cette tradition orale. De la compilation de tous ces rapports, surgissent des contradictions, des incompatibilités, que des Sages appelés les Amoraim gèrent en se référant à des sources tannaïques ${ }^{1}$.

Le régime de rationalité des Sages du Talmud ressemble à ce qu'Amossy (2014) a appelé un vivre-ensemble dans le dissensus. Mais pour ces Sages, l'étude dans l'incertitude et la multiplicité des opinions n'est pas un simple modus vivendi : c'est une raison d'être. Ils fondent leurs positions non seulement sur l'autorité de sources antérieures, mais aussi sur des règles herméneutiques précises (transmises depuis le Mont Sinaï), et sur l'acceptabilité des conclusions dans leur contexte économique et social. En surface, l'enjeu des discussions est la recherche des règles de comportement qui sont les plus appropriées à la tradition. Néanmoins, l'enjeu de fond est religieux : il s'agit de mettre le dialogue en œuvre pour tenter de retrouver le contenu originel du dialogue entre Moïse et Dieu, contenu dont les dissensus talmudiques tentent de prouver et de retrouver un sens premier univoque sans y parvenir. Le dialogue est donc à la recherche d'une Vérité absolue. Il ne s'agit pourtant pas d'un mode cartésien de contemplation rationaliste de la vérité à accepter telle quelle, sans discuter. BlumKulka, Blondheim et Hacohen (2002) ont bien montré la nature hautement dialectique des pages du Talmud, et le style agressif adopté dans la gestion des désaccords. Pourtant, l'école de Hillel, qui est prépondérante dans le Talmud, va jusqu'à faire des concessions à d'autres écoles, lorsque les arguments qu'ils avancent dans la discussion apparaissent comme plus convaincants. Cette caractéristique des discussions talmudiques a mené Fisch (2011) à suggérer que la rationalité des Sages du Talmud est dialogique. Cette rationalité est pratique; elle est ancrée dans des croyances religieuses, et dans une éthique existentielle.

Depuis la clôture de la rédaction du Talmud (au $6^{e}$ siècle), les centres d'étude - les Yeshivas - se donnent pour mission de recréer une chaine de tradition d'étude. Le texte talmudique est à la base de l'étude dans les Yeshivas. De plus, les pratiques de la Yeshiva invitent au dialogue, surtout la pratique de la chavruta - l'étude dyadique de textes talmudiques. Cet article est centré sur la nature de ces pratiques.

\section{Observations d'interactions dyadiques dans les Yeshivas}

\subsection{L'importance du texte talmudique dans la vie des Juifs pratiquants}

6 L'étude des textes talmudiques a toujours été considérée comme une activité centrale au sein de l'élite juive orthodoxe. Les maximes des Pères ${ }^{2}$ donnent un tableau détaillé du cursus du Juif pratiquant: "Cinq ans pour la Torah (la loi écrite), dix ans pour la Mishna (la loi orale), quinze ans pour le Talmud ». Bien sûr, à l'époque où la Mishna fut rédigée, le Talmud n'avait pas encore été écrit. Pourtant, l'activité qui consiste à commenter les paroles des Sages pour en inférer la Halakha - la conduite appropriée de la vie quotidienne, était déjà une pratique éducative. L'étude journalière est clairement définie: "Chacun doit toujours diviser ses jours en trois parties, un tiers pour les Écritures, un tiers pour la Mishna et un tiers pour le Talmud» (Traité Kiddushin, 34a). 
Rabenu Tam, le célèbre Tosafiste ${ }^{3}$, déclara : «maintenant, celui qui étudie le Talmud est exempt de l'obligation d'étudier les trois domaines, puisque le Talmud inclut des versets de la Bible, de la Mishna et de la Halakha (les règles de conduite) ainsi que des raisons et des explications raisonnables ". Ainsi, en étudiant le texte talmudique, l'apprenant continue les discussions de ses ancêtres (Weiss 1887). Rabbi Moshe Isserlis, le commentateur ashkénaze du Shulkhan Arukh ${ }^{4}$ le codex de la loi Juive - a décidé dans le même esprit que l'étude s'accomplit pleinement en se concentrant sur le Talmud. Pendant des siècles, l'élite de l'orthodoxie a suivi cette décision.

$7 \mathrm{Au}$ milieu du $20^{\mathrm{e}}$ siècle, un accord controversé entre David Ben Gourion (le premier dirigeant de l'État d'Israël) et le chef spirituel de l'ultra-orthodoxie, le Hazon Ich, permit d'ouvrir les portes des Yeshivas à tous les jeunes ultra-orthodoxes. L'élite des Yeshivas fut alors remplacée par une société d'apprenants (Friedman 2006).

8 Cet article porte sur l'étude de textes talmudiques dans les Yeshivas ultra-orthodoxes d'aujourd'hui. Celle-ci s'accomplit généralement au sein de paires d'apprenants qui sont appelées Chavrutas. L'apprentissage en Chavruta est une pratique récente: Stampfer (1995) a remarqué que dans la Yeshiva de Volozhin (19e siècle), le modèle de toutes les Yeshivas modernes, l'étude des textes sacrés se faisait souvent de façon individuelle. Ce fait est en opposition avec ce que stipulent les sources rabbiniques. Pourtant, le regain de popularité de l'apprentissage en Chavruta ne provient pas de la relecture de ces sources. La raison en est plutôt la transformation de la société ultraorthodoxe en une société d'apprenants dans laquelle sont également incorporés des étudiants moyens, voire médiocres. Dans cette situation, le dialogue dans les Chavrutas qui n'avait été qu'une forme possible, conseillée mais souvent non-pratiquée, devint indispensable. Cet article se focalise sur la nature du discours autour de textes talmudiques accomplie dans des Chavrutas.

\subsection{Questions de recherche}

Deux hypothèses raisonnables peuvent être avancées sur la nature des interactions entre étudiants de Chavrutas. La première est que les opinions divergentes qui apparaissent dans le Talmud sont des ressources qui encouragent l'expression d'oppositions et de défis. La seconde hypothèse est que les désaccords dans les sources textuelles sont des ressources pour une immersion collective et collaborative dans une pluralité d'opinions. Ces hypothèses sont toutes deux plausibles, tant les études empiriques sur l'apprentissage en Chavrutas sont rares: Holzer (2005) a proposé un modèle théorique de l'apprentissage en Chavruta ; Brown et Malkus (2006) se sont basés sur des interviews; Kent et Cook (2012) ont observé une Chavruta en dehors d'un contexte traditionnel. Les études conduites par Schwarz $(2011,2014,2015)$ dans une Yeshiva font pencher la balance du côté de la seconde hypothèse (la collaboration), mais les conclusions de ses travaux étaient basées sur un ensemble de données très restreint.

Un objectif central de notre recherche est d'identifier le régime de rationalité des apprenants dans les Yeshivas. Le manque d'études empiriques ne permet pas de proposer d'hypothèses sur cette question et nous ne savons pas si la rationalité dialogique qui caractérisait les discussions des Sages du Talmud (Fisch 2005) est effectivement redupliquée. Nos questions de recherche sont les suivantes : 
- Quels types d'interactions discursives sont produits dans l'étude de textes talmudiques en Chavruta?

- Quels processus discursifs sont impliqués dans de telles productions?

- Quel est le régime de rationalité de l'étude en Chavruta?

\section{Description du corpus de la recherche}

\subsection{La dyade}

11 Avi et Moshe (noms fictifs) ont reçu une éducation ultra-orthodoxe classique. Ils ont étudié dans des Yeshivas renommées. Ils consacrent leur vie à l'étude, et ne travaillent pas. Avi a 32 ans ; il est né aux USA, est marié et a cinq enfants. Il étudie dans un Kolel (un centre d'étude pour étudiants mariés qui est moins contraignant que la Yeshiva) depuis 11 ans. Moshe a 28 ans; il est né en Israël, est marié et a trois enfants. Moshe étudie dans le même Kolel depuis 7 ans. Avi et Moshe forment une Chavruta depuis 5 ans.

Le Kolel dans lequel Avi et Moshe étudient se trouve dans une localité proche de Jérusalem. Il comprend à peu près 50 étudiants expérimentés. Le programme d'études journalier inclut trois périodes d'étude ( sdarim»), matin, midi et soir. Pour chacune d'entre elles, les dyades changent, ainsi que le contenu de l'étude.

\subsection{La méthode d'apprentissage}

Les pratiques décrites dans cet article abordent différentes questions soulevées pendant l'étude de l'un des 39 travaux interdits pendant le Shabbat (le Sabbat): le travail de lavage. La méthode générale adoptée par les étudiants consiste à inférer la Halakha, ou code de conduite, de la tradition (elle est appelée Aliba dehilkheta). Elle stipule que l'étudiant oriente son étude des textes talmudiques vers des questions de comportements pratiques, plutôt que vers des questions théoriques. La dyade lit tout d'abord les passages pertinents du Shulkhan Arukh, puis s'efforce de comprendre le développement de la Halakha, depuis ses sources les plus anciennes - la Mishna et le Talmud, jusqu'aux législateurs qui les ont commentées - les rishonim et les akharonim ${ }^{5}$.

\subsubsection{Le thème du « lavage »}

Les thèmes dans le Talmud paraissent le plus souvent rébarbatifs aux yeux d'un lecteur non initié. Sans doute aurions-nous pu choisir un thème plus accessible - et il en existe dans le Talmud. Cependant, notre choix est justifié dans la mesure où il nous permet de tirer des conclusions sur le régime de rationalité de nos étudiants. Il s'agit de l'interdiction de laver pendant le Shabbat, car le lavage est considéré comme une sorte de « travail ».

15 L'étude commence par la lecture de sources halakhiques sur les préceptes ayant trait au lavage pendant le Shabbat dans le Shulkhan Arukh. Les clauses de ces sources portent sur trois sujets: (1) Le fait que celui qui secoue son manteau le Shabbat est passible d'offrande expiatoire (2) Le frottement [de la rigidité] d'un linge et (3) le nettoyage d'un vêtement sali par de la terre. Après avoir lu ces clauses, la Chavruta passe à des sources talmudiques concernant ces lois. 

(137a). La loi est applicable lorsque trois conditions sont remplies: le vêtement est nouveau, il est noir et son propriétaire est kapid (soigneux - il prend soin de la propreté de ses vêtements). Les discussions de la Chavruta se basent sur des discussions de rishonim (Rashi, Tosefoth, HaRosh, Ran, Rambam) sur deux sujets: (a) Quelle est la définition de kapid? et (b) est-ce que le fait de secouer un vêtement est inclus dans le domaine d'application des lois sur le « lavage »?

Le second sujet, «le frottement du manteau » apparait aussi dans le traité Shabbat (130a). Les Sages réfléchissent sur le type de vêtement (extérieur ou intérieur) qu'il est permis de frotter. La Chavruta tente de clarifier ce que veut dire le terme kapid, et discute des raisons qui mènent à frotter un vêtement le Shabbat.

Le second sujet fournit une source au troisième, qui apparait dans le Shulkhan Arukh sur les préceptes concernant le lavage - enlever de la boue d'un vêtement par l'intérieur du vêtement et non de l'extérieur. Les étudiants réfléchissent sur la façon dont Rashi comprend deux passages du Talmud qui semblent en contradiction.

\section{Méthodologie. Une étude de cas}

19 Bien que la méthode d'apprentissage en Chavruta puisse être à première vue traitée d'argumentative, elle est en réalité très particulière. Tout d'abord, le contexte d'apprentissage journalier avec le même compagnon d'étude pendant des périodes prolongées autour des mêmes textes n'est guère répandu en dehors du monde des Yeshivas. De plus, l'apprentissage en Chavruta n'a été que rarement étudié. Cette situation nous a amené à choisir la méthode de l'étude de cas, tout d'abord parce que l'apprentissage en Chavruta ne peut pas être facilement comparé à d'autres phénomènes (Eisenhardt \& Graebner 2007); et ensuite, parce qu'il se déploie dans son contexte naturel, et permet une thick description (MacDonald \& Walker 1977).

\subsection{Collection de données et analyse}

Avi et Moshe ont clarifié la question du lavage pendant trois semaines. Dix sessions ont été enregistrées. Les pratiques rapportées dans cet article ont été observées pendant trois jours consécutifs au cours de sessions qui ont duré 90 minutes chacune. Chaque session a été enregistrée sur bande audio par l'un des membres de la dyade. Les chercheurs étaient absents. Les enregistrements ont alors été transcrits, et analysés par ces mêmes chercheurs. Nous avons ainsi identifié des mécanismes sous-jacents à l'apprentissage en Chavrutas.

21 Nous décrivons ici quelques exemples qui manifestent des formes d'apprentissage spécifiques. Tous les exemples portent sur le sujet de « celui qui secoue son vêtement ». $\mathrm{Au}$ préalable, quelques informations générales aideront le lecteur à comprendre des discussions qui sont très pointues.

Exemple 1 : une relecture collaborative

Pour des étudiants dans un Kolel (qui ont donc déjà étudié pendant leur adolescence dans des Yeshivas), le Traité de Shabbat est bien connu. Il a été étudié au moins trois ou quatre fois, dont au moins une de façon approfondie. Réétudier des textes talmudiques est l'une des caractéristiques de l'apprentissage dans les Yeshivas et Kolels (Schwarz

Argumentation et Analyse du Discours, 25 | 2020 
2011). Dans le cas que nous avons observé, lorsque les étudiants ont lu le Traité, l'objectif de la relecture était la compréhension de la Halakha. La lecture a donc commencé avec des clauses du Shulkhan Arukh, et s'est poursuivie avec le Talmud en tant que source de ces clauses. Le protocole qui suit est un exemple de cette lecture. Chaque fois qu'un étudiant lit à voix haute, nous le signalons en italiques. La traduction a été faite par l'auteur.

1 Avi :Oui [un des étudiants commence à fredonner ${ }^{6}$ ] On a ici la première clause] celui qui secoue son manteau 2 Moshe : Quoi ? De quoi ça parle?

3 Avi : celui qui secoue de la rosée de son manteau

4 Moshe: Oui

5 Avi : Secouer [bruit de pages tournées]. On a la seconde clause celui qui enlève des choses de son manteau [bruits de pages tournées]. La troisième clause est pertinente? 6 Moshe : Celui qui fait attention à son vêtement

7 Avi : Le rouleau, on peut essorer ses vêtements [fredonne] le rouleau sur lequel ses vêtements sont mis. Je veux suivre l'ordre des clauses, d'accord? OK, donc tu vois que celui qui secoue ses vêtements commence à la fin du chapitre "le tonneau", Shabbat, 136. Rav Huna a dit celui qui secoue son manteau le Shabbat doit apporter une offrande expiatoire.

8 Moshe: Oui

9 Avi : Tu veux voir dans la Gemara?

10 Moshe : C'est écrit dans la Gemara.

11 Avi : Ouais euh? Bon, on voit ça.

12 Moshe : Tu l'as allumé ? [=l'enregistreur]

13 Avi : Oui, c'est là-bas en bas de Shabbat 136. Rav Huna a dit Rav Huna a dit celui qui secoue son manteau le Shabbat doit apporter une offrande expiatoire. Et ils n'ont parlé que de nouveaux vêtements, mais avec des vieux [vêtements [, y'a rien, y'a rien

14 Moshe : [fredonne]

15 Avi : Et ils ne parlent que de [vêtements] noirs

16 Moshe : Où c'est ? 13 ?

17 Avi : 136b

18 Moshe : 136 ?

19 Avi : 136b au bas de la page.

20 Moshe: Oui. Rav Yehuda a dit que Rav a dit que partout où les Sages ont interdits à cause des apparences, c'est interdit même dans l'intimité.

21 Avi : Oui.

22 Moshe : La Gemara à la page 136b en bas dit... elle commence avec la Mishna. C'est écrit dans la Mishna celui dont les vêtements sont tombés dans l'eau sur son chemin, marche avec eux, et ne s'en préoccupe pas

23 Avi : Qu'est-ce que tu veux dire par ne s'en préoccupe pas?

24 Moshe : Il les porte.

25 Avi : De quoi se préoccupe?

26 Moshe : C'est d'essorer, ça le préoccupe.

27 Avi : Quoi?

28 Moshe : D'essorer.

29 Avi : Quel est le problème s'il essore?

30 Moshe : S'il essore, y'a un problème de nettoyage, de piétinement, beaucoup de problèmes.

31 Avi : Alors, qu'est-ce qui est écrit ici ? Il arrive à la cour extérieure, il les étale au soleil mais pas devant tout le monde.

32 Moshe : C'est écrit qu'il les étale au soleil mais pas devant tout le monde. On voit qu'il y a une différence entre un endroit où les gens peuvent voir et un endroit où les gens ne peuvent pas voir.

33 Avi : OK. La Gemara dit [commence à fredonner] Rav Yehuda dit que Rav dit que partout où les Sages disent à cause des apparences, c'est interdit même dans l'intimité. 
34 Moshe: Il est enseigné: Il les étale au soleil mais pas devant tout le monde. Tu vois, c'est différent.

35 Avi : Mais tu dis que c'est une controverse entre Tannaïm. Parce qu'il est enseigné [dans une autre source mishnaïque] Il les étale au soleil mais pas devant tout le monde. Rav El'azar et Rav Shimon interdisent. Donc, en fait, on voit qu'il y a une controverse entre Tannaïm. Le Tanna dans notre Mishna l'a permis.

36 Moshe : Il les étale au soleil mais pas devant tout le monde. Rav El'azar et Rav Shimon interdisent. Pourquoi ? Ils pensent que partout où les Sages ont interdit à cause des apparences, l'interdiction subsiste même dans le privé.

37 Avi : Oui.

38 Moshe : Oui. Rav Huna a dit: Celui qui secoue son vêtement le Shabbat, doit apporter une offrande expiatoire. C'est déjà écrit à la page 137a au haut de la page.

39 Avi : Mais c'est pas lié à ce qu'on a appris maintenant.

40 Moshe : C'est lié.

41Avi : Ça a l'air d'un autre sujet, hein?

42 Moshe: Bon, on regarde. Celui qui secoue son vêtement le Shabbat doit apporter une offrande expiatoire.

Avi cherche les clauses qui ont trait au lavage dans le Shulkhan Arukh. En (3), il lit les premiers mots de la clause « celui qui secoue de la rosée de son manteau ». Avi suggère alors de consulter directement le Talmud (9). En (16) et (18), Moshe demande où Avi lit (19) et continue sa lecture (20). Il lit la Mishna, qui est la source de la Gemara (22), et explique (26) que la Mishna veut dire que si quelqu'un porte des vêtements humides, il peut les essorer même pendant Shabbat. A la question qu'Avi pose sur la sorte d'interdiction impliquée (29), Moshe donne plusieurs réponses (30). Après qu'Avi continue sa lecture de la Mishna sur les façons de traiter l'humidité des vêtements (31), Moshe infère de la formulation de la Mishna qu'il y a une distinction entre endroits publics et privés. Avi lit alors à haute voix une source amoraïque (33) qui contredit la source mishnaïque que la dyade vient de lire. Sa lecture à haute voix défie la lecture de Moshe qui réagit au travers de lectures réitérées de la source mishnaïque (34). Avi lit alors à haute voix la source de leur controverse, qui est une controverse entre Tannaïm (35). Moshe répond en lisant une source mishnaïque (38). Avi se plaint que ce que Moshe lit n'est pas pertinent par rapport au sujet de la discussion, car le nettoyage et le secouement n'impliquent pas nécessairement de l'eau et de l'humidité (39). Moshe (40) réagit en disant qu'il y a un lien. Avi proteste modérément (41). Moshe répond en continuant la lecture 42).

De prime abord, ce protocole montre tout simplement un exemple de lecture collective pendant laquelle Avi et Moshe lisent tour à tour des sources halakhiques et talmudiques et s'interrogent l'un l'autre sur leur sens. Pourtant, comme nous l'avons déjà remarqué, les deux étudiants sont familiers du Traité. La lecture des sources est donc une relecture à la lumière de clauses halakhiques. Elle inclut une lecture ordinaire $(1,7,13,20,22,33$, $34,35,36,38,42)$, des procédures d'organisation de la lecture $(5,6,7,9,1011,13,39)$, l'élaboration d'explications $(3,24,28,30,31,32,34,35,36)$, et de questions $(2,23,25,27$, 29). Cette lecture est ancrée dans la culture de la Yeshiva: les deux étudiants chantonnent pendant leur lecture $(1,13,14,33)$. De plus, leur lecture de clauses halakhiques puis de sources talmudiques pour comprendre comment les décisions ont été prises, les amènent à réaliser au tour 39 que la source talmudique qu'ils ont lue n'est pas pertinente.

Cette lecture a un caractère collaboratif : la lecture d'un des étudiants est suivie par les explications de son compagnon (31-32, 34-35) ou par ses questions (22-23). L'interaction 33-38 ajoute une autre dimension à la collaboration: Avi et Moshe lisent les textes 
comme s'ils étaient les porte-parole de leurs auteurs: Le «Il est enseigné: celui qui les étale au soleil mais pas devant tout le monde » (34) qui est suivi par l'explication " Tu vois, c'est différent ", suggère que Moshe voit dans ce qui est écrit dans la Mishna une confirmation de ce qu'il pense. La réaction élaborée en (35) « mais tu dis que c'est une controverse entre des Tannaïm. Parce que c'est enseigné [...] on voit qu'il y a une controverse entre Tannaïm. Le Tanna dans notre Mishna l'a permis » est la réponse d'Avi à Moshe par le biais de la lecture d'une autre source mishnaique. Lorsque Moshe lit et commente "Rav El'azar et Rav Shimon interdisent. Pourquoi ? Ils pensent que partout où les Sages ont interdit à cause des apparences, l'interdiction subsiste même dans l'intimité » (36), ils complètent ce que des Amoraïm ont dit par le biais de Tannaïm. En d'autres termes, ils collaborent pour continuer la chaîne des interactions entre Sages et compléter leurs préceptes. collabortives, bien qu'ils soient omniprésents. Mentionnons simplement une enquete (aimplement une enquête typique, qui demande si enlever la boue d'un vêtement est possible le Shabbat. Cet acte ne peut pas être fait directement en grattant le vêtement, mais seulement en agissant de l'intérieur du vêtement, de manière que la saleté tombe «du dehors». Bien que cette description soit claire, les exégètes ne donnent pas une définition précise de cet acte. Enlever la boue peut avoir plusieurs sens qui oscillent entre laver et nettoyer. La loi stipule qu'il est interdit d'enlever la saleté de l'extérieur si la partie sale est de la boue. Pourtant, le Shulkhan Arukh n'utilise pas le terme kiskusune sorte de - (כיסכוס) frottement, qui apparait dans le Talmud mais le terme shifshufqui signifie (שפשוף) frotter. L'investigation qu'Avi et Moshe conduisent est empirique. Leur collaboration est non seulement soutenue par leur dialogue mais aussi par leurs actions : ils testent leurs suppositions en salissant le pantalon d'Avi, en le secouant, en le frottant et en regardant les conséquences de leurs actions. Chacun fait des expériences pour prouver qu'il a raison. A la fin de l'investigation, Moshe se rallie au point de vue d'Avi selon lequel le kiskus est défini par ce que cette action fait sur le pantalon.

Les textes talmudiques rapportent des discussions critiques qui sont conformes à un modèle pragma-dialectique de l'argumentation (van Eemeren \& Grootendorst 2003). dans lesquelles les oppositions sont nombreuses et leur gestion est agressive. Que se passe-t-il dans l'étude en Chavruta? Le discours y est-il argumentatif dans le même sens?

Les trois premiers exemples ont fourni une réponse négative : nous avons montré des cas de relecture collective, de co-construction, et d'investigation collective qui n'ont pas dévoilé de délibérations dans lesquelles les protagonistes émettent des opinions divergentes. Ceci montre que l'apprentissage en Chavruta n'est pas exclusivement argumentatif. Dans les deux derniers exemples que nous présentons, nous décrivons deux formes argumentatives, qui semblent spécifiques au contexte de la Chavruta. Le premier illustre ce que nous croyons caractéristique des discussions en Chavruta - des tentatives d'argumentation collective sous la contrainte de textes d'autorité. Nous rencontrons de telles tentatives lorsque notre dyade lit un texte talmudique dans le Traité de Shabbat 137a :

44 Moshe: Alors, celui qui secoue son manteau pendant le Shabbat doit apporter une offrande expiatoire. Et ils ont dit ça que pour des nouveaux vêtements mais quand ils sont pas nouveaux, y'a rien, et ils ont dit que pour les vêtements qui sont noirs, blancs, et rouges, y'a rien.

Argumentation et Analyse du Discours, 25 | 2020 
45 Avi : [baille] mais pour les blancs et les rouges [chantonne] y'a rien.

46 Moshe : Qu'est-ce qui est écrit ici ? Quelqu'un ici secoue. Qu'est-ce qu'il secoue? Rashi explique qu'il secoue de la poussière du vêtement.

47 Avi : Rashi. Celui qui secoue la poussière du manteau.

48 Moshe : Il doit apporter une offrande expiatoire, c'est ce que dit la Gemara. Il y a quelques restrictions.

49 Avi : Euh, pourquoi devrait-il l'apporter?

50 Moshe : La première chose est que -

51 Avi : Pourquoi devrait-il apporter une offrande ? Rashi dit que c'est parce que c'est son nettoyage. Qu'est-ce qu'il veut dire, c'est -

52 Moshe : Ça veut dire que c'est le nettoyage.

53 Avi : C'est le nettoyage

54 Moshe : - il le nettoie.

55 Avi : Qu'est-ce ça veut dire «c'est son nettoyage »? Rashi aurait dû dire [c'est la transgression $\mathrm{du}$ ] travail de nettoyage.

56 Moshe : Non.

57 Avi : C'est son nettoyage.

58 Moshe: Il y a ici quelque chose de nouveau. Ça veut dire que tu poses une question. Tu demandes qu'est-ce que c'est que « c'est son nettoyage » Dis à la place qu'il est coupable à cause du [travail de] nettoyage. Qu'est-ce ça veut dire, «c'est son nettoyage»? Il est possible d'arguer que c'est nouveau. Quelle est la nouveauté ? La nouveauté est qu'il est possible de nettoyer sans eau.

59 Avi : Ça veut dire que le sens littéral [de nettoyage] -

60 Moshe : - est nettoyage avec de l'eau, nettoyage avec eau.

61 Avi : Laver. Pourquoi tu comprends que le sens littéral de nettoyage est que c'est sans eau?

62 Moshe : C'est comme ça que je comprends.

63 Avi : C'est ce que pensent les enfants dans les crèches.

64 Moshe : Et c'est comme ça que le nettoyage marche.

65 Avi : Euh?

66 Moshe : C'est comme ça que les gens lavent, ils lavent avec de l'eau

67 Avi : Il y a beaucoup de manières de laver

68 Moshe : Et ben, les Tosfoth sont pas d'accord [avec Rashi]

69 Avi : Quoi ?

70 Moshe : Les Tosfoth disent que le nettoyage ne se fait pas qu'avec de l'eau parce que c'est la manière de laver, parce que quelquefois, on n'a pas besoin d'eau et ici, tu l'enlèves en secouant, et c'est donc un nettoyage

31 Moshe et Avi commencent leur lecture de la Gemara: Moshe introduit le sujet en lisant le commentateur Rashi (46), qui explique qu'il est illicite de secouer dans le cas où ce qui est sale est de la poussière. Avi exprime son accord en lisant Rashi à voix haute (47). Moshe continue la lecture de la Gemara (48) pour examiner les cas pour lesquels des offrandes expiatoires doivent être apportées. En contraste, Avi tente de déchiffrer la formulation étrange de Rashi - "c'est son nettoyage » au lieu de la formulation plus naturelle "c'est la transgression du travail de nettoyage " (Avi 51, 53, 55). Moshe ne comprend pas ce qu'Avi déclare : la formulation "c'est son nettoyage " signifie que le nettoyage peut se faire sans eau, bien que le sens littéral de nettoyage soit un lavage avec de l'eau (60). Avi s'oppose à cette affirmation, et demande à Moshe pourquoi il pense cela (61). La réponse de Moshe - le fait que c'est une supposition acceptée par tous (62) - est rejetée (63). Le désaccord entre Moshe et Avi continue (64-70) et se termine par le soutien que Moshe trouve dans la lecture des Tosfoth.

Cet exemple renforce ce qui constituait pour nous une hypothèse de départ: l'argumentation dans la Chavruta est contrainte par les arguments émis par des commentateurs. Pour émettre des arguments personnels, on doit trouver un 
commentateur de référence. Lorsque Moshe tente d'argumenter contre Avi - en fait, contre Rashi, il enrôle un de ses antagonistes traditionnels, les Tosfoth, mais il échoue. En effet, les Tosfoth n'abondent pas dans le sens que Moshe aurait souhaité, et l'argumentation prend fin. En d'autres termes, les arguments qui comptent dans l'argumentation de la Chavruta sont les arguments exprimés par les commentateurs traditionnels. Incontestablement, l'identification de leur orientation argumentative est une affaire d'interprétation - personnelle ou institutionnalisée au travers de commentateurs postérieurs qui ont établi des voies devenues traditionnelles pour comprendre les commentateurs classiques. Si l'argumentation en Chavruta est contrainte par la littérature exégétique que les apprenants ont sous la main, les arguments écrits par les commentateurs sont aussi des outils pour l'étudiant hésitant qui cherche à s'exprimer.

Dans l'extrait que nous avons décrit, Moshe n'a pas trouvé de soutien pour alimenter sa propre argumentation. Nous allons décrire comment il va réussir à initier une argumentation plus fructueuse.

Le cinquième exemple poursuit le quatrième. À la fin de ce dernier, Moshe lit les Tosfoth, qui expliquent qu'il y a diverses façons de nettoyer des vêtements, avec ou sans eau. À la fin de leur étude journalière, Avi et Moshe consultent ensemble plusieurs législateurs et y distinguent deux groupes : pour Rashi, le Rosh et Maïmonide, le nettoyage peut se faire sans eau; pour Tosfoth et la majorité des législateurs qui viennent après eux, le nettoyage ne peut se faire qu'avec de l'eau. Avi profite de la session qui suit pour clarifier ce qui se cache derrière cette controverse :

1065 Avi : Donc Rashi veut dire qu'il y a une autre distinction -

1066 Moshe : - Donc, c'est la réalité, c'est la réalité, c'est [sur quelque chose]

1067 Avi : [c'est la réalité]

1068 Moshe : D'une certaine manière, ça reste. C'est de la boue. Juste il secoue la

boue, et la tache reste. C'est normal.

1069 Avi : OK.

1070 Moshe : Alors, dans cette situation [il n'y a pas] [bâillement]

1071 Avi : OK, [dans cette situation] vraiment, de nettoyage.

1072 Moshe : De quoi me considères-tu comme responsable? Du résultat? Il n'y a rien parce qu'il voit que c'est resté. Il est responsable de son action qui est l'action de nettoyer.

1073 Avi : OK.

1074 Moshe : L'action de nettoyer ne se fait qu'avec de l'eau.

1075 Avi : [Comment on peut considérer quelqu'un responsable] pour des effets ? Tu es sur la souguya ${ }^{7}$ en (page) 137

1076 Moshe : Dans notre souguya, le vêtement était sale et il est devenu propre. C'est vrai qu'il n'y avait pas d'eau mais il y avait une action de nettoyage. Ouais, je dis qu'il y a une action de nettoyage. Oui, je dis que pour chaque travail, y'a deux choses. Y'a l'action, le travail [et y'a les effets du travail].

1077 Avi : [mais il y a une responsabilité] attachée à l'action. Tu n'es jamais responsable. Je te dis, si je joue un tour magique et je lave, je ne suis pas responsable, pas vrai? On n'a jamais vu que quelqu'un soit responsable à cause des effets. Qu'est-ce que tu veux dire, que dans notre souguya, je suis responsable parce que les vêtements sont propres?

1078 Moshe : [baille] ouais.

1079 Avi : Où, où, où est-ce que tu vois dans la Torah que quelqu'un est responsable [pour, pour]

1080 Moshe : [Parce que c'est ton action] C'est ton action. 
$\mathrm{Au}$ début de ce protocole, Avi prétend que si Rashi distingue entre une situation dans laquelle le vêtement garde un peu de saleté et une situation dans laquelle il est totalement propre, il devrait le dire explicitement (1065). Avi répond que Rashi n'est pas obligé d'être explicite car la réalité fournit une preuve qui soutient son argument selon lequel des taches peuvent rester ou ne pas rester $(1066,1068)$. Avi réagit en déclarant que dans cette situation incertaine, il y a tout de même une sorte de nettoyage (1071). Moshe l'interrompt pour dire qu'il est impossible d'imposer une sanction en fonction des effets simplement parce que le vêtement n'est pas totalement propre et celui qui le nettoie est responsable $(1072,1074)$. Moshe continue et détaille son approche: pour qu'une personne soit passible de sanction pour nettoyage, une action et son effet sont nécessaires (1076). Avi n'est pas d'accord sur l'idée que l'effet (la propreté ou la saleté d'un vêtement) puisse être déterminant (1077). Il n'arrête pas de contredire Moshe en affirmant qu'il n'existe aucun principe qui ferait de l'effet un critère déterminant (1079). Moshe continue sa ligne de raisonnement qui intègre l'action et son effet (1080). En comparaison avec l'exemple 4, l'argumentation est ici beaucoup plus sophistiquée. Ils comprennent que suivant Rashi, c'est l'effet de l'action de nettoyage qui compte. Pour les Tosfoth, l'action compte ainsi que son effet. Pourtant, Avi et Moshe ne s'en tiennent pas aux mêmes arguments que leurs ancêtres. Les étudiants reviennent deux jours après à la définition d'une action et de ses effets. Jusque-là, ils ont été assez analytiques. Nous allons voir qu'ils vont choisir leur camp ils vont commencer à s'identifier avec un des camps de la controverse.

1607 Avi : [Je crois, je crois] qu'on doit faire une distinction entre Rashi et les Tosfoth. Les Tosfoth disent que secouer de la poussière, c'est rien. Rashi dit que secouer pour enlever la poussière, c'est quelque chose. C'est là, la controverse. C'est comme ce qu'on voit (cette controverse) dans le Biur Halakha. Allez, on commence avec ce sur quoi on est d'accord -

1608 Moshe : Quelle est sa question?

1609 Avi : Allez, on commence avec ce sur quoi on est d'accord. On est d'accord qu'il y a un concept de nettoyage même sans eau. Comme ce qu'on a vu avec kiskus.

1610 Moshe: Qu'est-ce que c'est kiskus ? C'est prendre un morceau de tissu et le frotter -

1611 Avi: - et le frotter.

1612 Moshe : Deux côtés, l'un contre l'autre.

1613 Avi : Oui.

1614 Moshe: Le Rosh l'admet aussi. Pourquoi il dit que c'est une action de frottement?

1615 Avi : Aïe Aïe [Claquement de mains]

1616 Moshe : Pourquoi secouer ici?

1617 Avi : Y'a pas de nettoyage sans eau? Pas de nettoyage, comment ça marche avec cette phrase des Tosfoth et des rishonim qu'il n'y a pas de nettoyage sans eau? [trois coups sur la table]. Qu'est-ce que tu dis?

1618 Moshe : Suivant la Torah, suivant les Sages, je ne sais pas.

1619 Avi : [baille] Ah.

Dans cet extrait, Avi tente de comprendre la controverse entre Rashi et les Tosfoth. Il commence par ce qui semble indiscutable. Suivant Rashi, secouer la poussière de vêtements est considéré comme du nettoyage; selon les Tosfoth, cela ne l'est pas (1607). Avi essaye de développer ce point pour affirmer que Rashi aussi bien que les Tosfoth sont d'accord qu'il n'y a pas d'action de lavage sans eau, comme c'est le cas du kiskus (sorte de frottement) qui n'est pas une action $^{8}$ (1609). Moshe clarifie une fois de plus ce que kiskus est, et arrive à un accord avec Avi (1610-11): il y a une sorte de frottement vigoureux qui est interdit même sans eau. Moshe expose sa position qui 
pose un dénominateur commun entre le kiskus et le nettoyage, qui sont tous deux des actions (1614): secouer n'est pas une action réelle, et est donc permis (1616). Avi répond qu'il n'y a pas de véritable nettoyage sans eau, et s'interroge sur la question de savoir comment on peut interdire le nettoyage sans eau (1617). Moshe suggère avec hésitation une distinction entre laver avec de l'eau, qui est interdit par les Écritures, et laver sans eau, qui est interdit par les Sages.

Cet extrait montre plus d'engagement personnel de la part de la Chavruta que dans les extraits précédents. Nous avons déjà observé l'identification d'arguments textuels en conflit, et les essais infructueux pour les incorporer dans l'argumentation de la dyade (44-70). Le dernier extrait que nous introduisons ici est lié aux principes qui régissent cette controverse. Les voix des deux apprenants se fondent dans les voix de leurs ancêtres, et ces voix s'entremêlent :

1864 Moshe : Il est interdit de faire un kiskus à un vêtement. Secouer est interdit pour Rashi et permis pour les Tosfoth. Qu'est-ce qui se passe avec le secouement quand toutes les conditions sont remplies ? Pourquoi c'est permis pour les Tosfoth? 1865 Avi : C'est le problème du changement entre kiskus et secouer. C'est différent pour Rashi et les Tosfot.

1866 Moshe : Les Tosfoth disent que le kiskus est une action. Et pour secouer, y'a pas d'action, donc c'est permis. Si tu enlèves de la poussière, ça t'est égal que tu enlèves de la poussière

1867 Avi : Qu'est-ce que ça veut dire, pas d'action? Qu'est-ce que ça veut dire, pas d' action?

1868 Moshe : Parce qu'il n'y a pas d'action. Rashi dit que même si [fait un geste de secouement] il n'y a pas action, ça enlève la poussière

1869 Avi : On ne disait pas, il y a quelques jours, que les effets, ça marche pas avec les Tosfoth ? Les effets, ça marche avec Rashi

1870 Moshe : Secouer est une action

1871 Avi: Un bon point. Y'a une action. Alors, qu'est-ce que les Tosfoth nous veulent?

1872 Moshe : ...c'est avec les mains mais tu l'enlèves pas le (.) avec les mains tu secoues et ça part tout seul.

1873 Avi : Ah, on a vu que le tremper, c'est le laver. Il y a la une situation où tu fais quelque chose sans faire d'action. Tu le trempes, hein, et, et, tu commets l'action interdite de laver. C'est ça, hein? Tremper, c'est laver, c'est ce que disent les Tosfoth. Ce n'est que si tu le fais avec de l'eau, quand tu le fais sans autre action qui crée une situation de propreté. Secouer, secouer ne suffit pas. C'est pas une action sur le vêtement.

Pour Moshe, l'action de kiskus consiste en un frottement très vigoureux. Il s'ensuit que même si le résultat de cette action ne conduit pas à la suppression d'une tache et laisse des marques, celui qui accomplit cette action transgresse l'interdiction du travail de nettoyage le Shabbat. Donc, Moshe se demande pourquoi les Tosfoth ont autorisé l'action de secouer. Avi répond à la perplexité de Moshe (1865) en mentionnant que pour les Tosfoth, secouer est permis parce qu'ils sont en désaccord avec Rashi qui croit que l'action qui consiste à secouer altère le vêtement. Moshe tombe d'accord avec Avi (1866) sur le fait que les Tosfoth ne rendent pas celui qui secoue responsable car il n'y a pas d'action, même dans le cas où l'effet du secouement est que le vêtement est propre. Avi exprime sa confusion (1867) sur l'assertion des Tosfoth. Moshe répond (1868) que ce qui détermine s'il y a ou non transgression est l'action, et pour Rashi le fait de secouer est une transgression car le critère de ce qui est considéré comme une transgression est son effet. Avi (1869) avoue alors que ce qui n'était pas clair pour lui jusqu'à présent, était que le critère de l'effet ne correspond pas avec l'interprétation de la Gemara par 
les Tosfoth. Sa formulation ici ( On voulait pas dire ça il y a quelques jours ») ne laisse aucun doute sur le fait que sa réflexion a progressé entretemps, elle est la concrétisation d'une idée qui était restée inarticulée. Moshe est d'accord avec cette partition d'opinions entre Rashi et les Tosfoth (1870). Pourtant, il objecte que si pour les Tosfoth, l'action détermine s'il y a transgression ou non, comme secouer est bien une action, on peut difficilement l'autoriser. Moshe initie alors une explication (1871), qui est complétée par Avi (1872) : secouer un vêtement n'est pas vraiment une action, car l'acte n'est pas opéré directement sur le vêtement. Il est remarquable qu'à la fin de leur interaction, après avoir réalisé que leur désaccord réactive une controverse entre Rashi et les Tosfoth, ils s'entraident pour comprendre ce qui a été écrit par un des protagonistes (les Tosfoth) et qui met en question la cohérence du point de vue de Moshe. Avi, qui s'oppose à l'argument tenu par Rashi, ne voit même pas cette incohérence comme susceptible de renforcer son propre argument.

Nous avons vu qu'à la fin de leur interaction (et en fait, à la fin d'une série de discussions), la Chavruta devient consciente du fait que le désaccord entre ses partenaires est un désaccord entre deux exégètes, Rashi et les Tosfoth. L'approche que Moshe défend à propos du rôle déterminant de l'action est soutenue par les Tosfoth, alors que l'approche qu'Avi adopte, suivant laquelle les effets de l'action sont déterminants, est soutenue par Rashi. Il est bien clair que lorsqu'Avi et Moshe ont parcouru les décisions des législateurs classiques au cours du troisième jour de leurs discussions, ils sont devenus conscients du fait que le problème de l'action et de ses effets était au cœur des divergences d'opinions entre ces législateurs. Pourtant, leur impression restait inarticulée, et devait se concrétiser par le biais de la reconstruction d'une controverse entre législateurs - entre Rashi et les Tosfoth. Ils ont non seulement identifié leur voix avec eux (Avi avec Rashi et Moshe avec les Tosfoth), mais ont entremêlé ces voix dans leur argumentation.

La coexistence des voix d'Avi et de Moshe et de ceux auxquels ils ont insufflé de la vie les voix de Rashi et des Tosfoth - rappelle inévitablement des notions bakhtiniennes. Dans The Dialogic Imagination (1981), Bakhtine introduit les concepts d'hétéroglossie et de dialogisme, pour expliquer la génération du sens au travers de la "primauté du contexte sur le texte » (hétéroglossie), la nature hybride du langage (polyglossie) et la relation entre énonciations (intertextualité). Pour Bakhtine, exprimer une énonciation signifie « s'approprier des mots des autres et les peupler avec une intention propre ». En tant que critique littéraire, Bakhtine avait perçu le dialogisme inscrit sur le mode intentionnel dans des chefs-d'œuvre de la littérature. Nous suggérons que, comme des écrivains, nos étudiants de Chavruta se sont fondés sur des textes anciens pour donner naissance à leurs voix propres et ont intentionnellement ajouté leurs voix au chœur des voix réanimées de leurs Pères.

\section{Discussion}

41 Nous avons identifié plusieurs phénomènes dans l'étude de textes en Chavruta: (re)lecture collaborative, co-construction, enquête collaborative, argumentation sous les contraintes de sources d'autorité, et identification avec des commentateurs qui argumentent entre eux. Tous ces phénomènes ont trait à deux types d'activités, collaboration et argumentation, dont la combinaison dans l'étude dyadique de textes talmudiques est remarquable. 

aux Maîtres (exégètes ou législateurs) qui ont utilisé le texte talmudique pour en tirer des conclusions halakhiques. Les protocoles que nous avons présentés ont trait au «travail » de lavage. Les décisions qui apparaissent dans la littérature rabbinique ne dévoilent que rarement les principes qui les régissent. Il semble qu'aujourd'hui (et probablement depuis le $19^{\mathrm{e}}$ siècle), les apprenants cherchent à trouver de tels principes sous-jacents. L'apprenant qui découvre un principe qui correspond à ce que le Maître exégète ou législateur - commente ou décide, adopte sa voix. Le premier exemple en foisonne. Le second exemple (co-construction) manifeste aussi les efforts des apprenants pour trouver des principes dans le texte qu'ils lisent (par exemple, l'intensité de l'action ou l'intention de celui qui agit), mais, dans cet exemple, les autorités rabbiniques qui ont écrit le texte ne sont pas considérées. Nous suggérons que cette quête de principes aide la Chavruta à trouver des stratégies pour justifier leurs positions au sein d'un système d'autorité basé sur une chronologie (Tannaïm, Amoraïm, Rishonim, Akharonim).

Comme la co-lecture collaborative et la co-construction, l'investigation présentée dans le troisième exemple est collaborative au sens fort, en ce que l'attention d'Avi et Moshe était toujours partagée et leurs actions toujours coordonnées. Les apprenants ont sali 
leurs pantalons intentionnellement, les ont frottés avec ou sans eau, vigoureusement ou non, et ont observé les résultats de leurs actions.

Le troisième mécanisme qui est omniprésent dans les exemples 4 et 5 est l'identification progressive avec des figures d'autorité (exégètes ou législateurs). Dans le quatrième exemple, Moshe et Avi utilisent des arguments exprimés par Rashi et les Tosfoth dans leur propre argumentation. Ces arguments, qui sont a priori des contraintes, sont des ressources pour ces apprenants hésitants. Si pour l'exemple 4, Moshe et Avi n'arrivent pas à articuler une argumentation cohérente, l'exemple 5 montre au contraire qu'ils jouent avec succès les rôles de Rashi et des Tosfoth. La prise de conscience $d u$ fait qu'ils représentent les raisonnements tenus par Rashi et les Tosfoth n'est pas immédiate, mais dans le dernier protocole de l'exemple 5, leur argumentation devient extrêmement vivante, et entremêle leurs voix qui font écho à celles de Rashi et des Tosfoth.

Nous avons montré la nature collaborative-argumentative de l'étude en Chavruta. Adoptons pour un moment une attitude talmudique : Cette étude, est-elle vraiment collaborative-argumentative? L'émergence et le raffinement des arguments, leur passage d'un interlocuteur à l'autre, ressemblent à des coups de pied dans une balle sur un terrain de jeu. Une meilleure manière de définir ce que nous avons vu est de parler d'une collusion (du latin ludere - jouer) espiègle : nos interlocuteurs participent à un jeu qui leur permet de maintenir une tradition. Ils ont suivi des règles traditionnelles comme, par exemple, ne rien dire de nouveau, ils se sont adaptés au présent et ont ouvert la voie à des significations futures que d'autres Chavrutas pourraient remodeler dans le présent et le futur. Ils ont contribué à renforcer la constitution d'une communauté d'apprenants.

Comment peut-on décrire la rationalité de nos apprenants? D’un côté, Avi et Moshe ont tenté de se conformer aux règles de la pragma-dialectique dans leur argumentation, ont déployé des interactions remarquablement dialogiques, et ont de plus obéi aux contraintes des autorités rabbiniques. D'un autre côté, ils ont exercé leur liberté en choisissant les autorités rabbiniques qui soutiennent leur voix dans le dialogue. Pourtant, le texte talmudique décrit des dialogues qui s'ouvrent à toute la société : le Sage du Talmud s'entretient non seulement avec un autre Sage mais aussi avec un marchand ou un voleur, avec un collecteur d'impôts ou un fermier, avec un procureur romain ou une prostituée, pour apprendre quelque chose d'eux. Nos étudiants, en revanche, sont enfermés dans des thèmes aujourd'hui obsolètes qui leur sont imposés, et ne parlent qu'à ceux qui leur ressemblent. Leur argumentation, qui a trait a priori à la loi juive, n'a pas de répercussion pratique, puisqu'ils doivent se conformer à des décisions qu'ils ne peuvent pas mettre en question. Pourtant, si la communauté d'apprenants qu'ils contribuent à former ne reflète pas la rationalité dialogique et pratique qui apparait dans le texte talmudique, leur quête de vérités par le biais de discussions critiques autour de textes qui invitent à les découvrir, révèle une rationalité qu'ils considèrent comme leur contribution au prolongement d'une révélation divine. 


\section{BIBLIOGRAPHY}

Amossy, Ruth. 2014. Apologie de la polémique (Paris : PUF)

Bakhtin, Mikhail. 1981. The Dialogic Imagination: Four Essays. Ed. Michael Holquist. Trans. Caryl Emerson \& Michael Holquist (Austin \& London: U. of Texas Press)

Blum-Kulka, Shoshana, Menahem Blondheim, Gil Hacohen. 2002. «Traditions of dispute: from negotiations of Talmudic texts to the arena of political discourse in the media ", Journal of Pragmatics 34, 1569-1594

Brown, Steven, Mitchel Malkus. 2007. « Hevruta as a form of cooperative learning », Journal of Jewish Education 73 : 3, 209-226

Eisenhardt, Kathleen, Melissa Graebner. 2007. « Theory building from cases: Opportunities and challenges ", The Academy of Management Journal, 50 :1, 25-32

Fisch, Menachem. 2005. The dialogical rationality of Judaism's formative canon (Jerusalem: Shalom Hartman Institute)

Fischer, Shlomo. 2009 [1989], « Jewish Salvational Visions, Utopias, and Attitudes Towards the Halacha ", Adam B. Seligman (Ed.), Order and Transcendence: The Role of Utopias and the Dynamics of Civilizations (Leiden: Brill), 62-75

Friedman, Menahem. 2006. «'For the miracles': The prosperity of the Torah world (Yeshivot and Kollels) in Israel ». I. Etkes (Ed.). Yeshivot and Battei Midrash (Jerusalem: The Zalman Shazar Center for Jewish History), 431-442

Holzer, Elie. 2006. « What connects "good" teaching, text study and hevruta learning? A conceptual argument », Journal of Jewish Education 72 :3, 183-204

Kent, Orit, Allison Cook. 2012. « Havruta Inspired Pedagogy: Fostering An Ecology of Learning for Closely Studying Texts with Others », Journal of Jewish Education 78, 227-253

MacDonald, Barry, Rob Walker. 1977. « Case Study and the Social Philosophy of Educational Research », D. Hamilton et al. (eds). Beyond the numbers Game. London: Macmillan Education

Merriam, Sharan. 1998. Qualitative Research and Case Study Applications in Education (San Francisco: Jossey-Bass)

Schwarz, Baruch. 2011. « 'Hevruta' learning in Lithuanian Yeshivas: recurrent learning of Talmudic issues », I. Etkes, T. El'or, M. Heyd \& B. B. Schwarz (Eds). Education and Religion: Authority and Autonomy (Jerusalem: Magness Publishing House), 279-308

Schwarz, Baruch. 2014. « Authoritative or authoritarian voices in traditional learning in Jewish institutions ", A. Iannacone \& T. Zittoun (Eds), Activities of Thinking in Social Spaces (Hauppauge, NY : Nova Science Publishers), 131-148

Schwarz, Baruch. 2015. « Discussing argumentative texts as a traditional Jewish learning practice », L. B. Resnick, C. S. C. Asterhan \& S. Clarke (Eds), Socializing Intelligence Through Academic Talk and Dialogue (Washington DC: AREA Books), 153-162

Stampfer, Shaul. 1995. The Lithuanian Yeshiva in its emergence (Jerusalem: Merkaz Zalman Shazar) [en hébreu]

Urbach, Ephraim. 1970. Baale Hatosafoth (Jerusalem: Mosad Bialik) [en hébreu] 
van Eemeren, Frans, Rob Grootendorst. 2003. A Systematic Theory of Argumentation: The Pragma-

Dialectical Approach (Cambridge : Cambridge U. P.)

Weiss, Isaac. 1887. Dor Dor Vedoreshav [Each generation and its Scholars]. Vol. 3 (Wien)

\section{NOTES}

1. Les Tannaïm sont des Sages dont les préceptes ont été compilés dans la Mishna (au $3^{\mathrm{e}}$ siècle de l'ère commune). Après cette compilation, d'autres Sages, les Amoraim, ont délibéré sur la Mishna. Ces délibérations se trouvent dans la Gemara. Le Talmud inclut la Mishna et la Gemara.

2. Les Maximes des Pères (Pirkei 'Avot) rassemblent les paroles de sagesse des plus importants rabbins de Judée entre le $2^{\mathrm{e}}$ siècle avant l'ère commune et le $3^{\mathrm{e}}$ siècle de l'ère commune.

3. Les Baalei HaTosafoth (aussi appelés Tosfoth) sont des Sages médiévaux qui ont commenté le Talmud. La majorité d'entre eux sont du nord de la France et de l'Allemagne. Ils ont souvent travaillé sur les interprétations de Rashi sur le Talmud en les critiquant, voire en les réfutant. Les Tosfoth sont aussi considérés comme des législateurs. Ils adoptent une approche critiquedialectique, et Rabbenu Tam est un chef de file de cette approche (Urbach 1970).

4. Le Shulkhan Arukh est le code légal Juif le plus connu. Son auteur, Joseph Caro, l'a écrit à Safed (aujourd'hui en Israël) en 1563.

5. Les rishonim sont les premiers législateurs qui ont écrit leurs décisions avant l'apparition du Shulkhan Arukh (en 1563). Les akharonim sont les législateurs qui ont écrit leurs décisions après le Shulkhan Arukh. Il y a une stratification d'autorité très claire entre les rishonim et les akharonim, et un akharon (et a fortiori un étudiant) ne s'oppose à un rishon qu'à la condition que son opposition soit soutenue par un autre rishon.

6. La lecture à haute voix du Talmud est souvent accompagnée d'un fredonnement, ou d'une cantillation, comme une prière.

7. Le terme "souguya» signifie "problème" dans la culture talmudique. Typiquement, une souguya est traitée dans de nombreux endroits du Talmud. Pour comprendre une souguya, l'apprenant doit donc s'engager dans des activités qui impliquent la comparaison de différentes sources.

8. Le mot «action » qu'utilisent les étudiants se réfère à un agir dans le contexte du Shabbat. Dans le dialogue, une question centrale est si épousseter, secouer ou frotter peuvent être des agir dont l'accomplissement est potentiellement questionnable pendant le Shabbat.

\section{ABSTRACTS}

The present study offers a systematic analysis of talk practices of ultraorthodox Jews learning Talmudic texts in Yeshivas - traditional Houses of Study, in a dyadic setting called Chavruta. We investigate about the nature of these talk practices and about the kind of rationality which deploys in Chavruta learning. Analyses of the Talmudic text already stressed its highly dialectical character and the dialogic rationality that characterizes the discussions among Sages of the Talmud. We observe here two experienced students learning a Talmudic theme in a Chavruta setting in consecutive sessions. We identify various forms of collaborative argumentation in their learning, and show that although the dialogic and practical rationality that appears in the 
Talmudic text are missing in the Chavruta setting, the quest for truth of the learners around the Talmudic text reveals a rationality anchored in religious beliefs and in existential ethics.

Cette étude offre une analyse systématique des pratiques discursives de deux Juifs ultraorthodoxes qui étudient des textes talmudiques dans une Yeshiva - un centre d'étude traditionnel - dans un contexte dyadique appelé la chavruta. Des analyses du texte talmudique ont déjà mis en lumière le caractère hautement dialectique et la rationalité dialogique qui caractérisent les discussions entre Sages du Talmud. Nous explorons ici la nature de l'argumentation mise en œuvre par deux étudiants expérimentés qui étudient un thème talmudique au cours de sessions successives, et cherchons à identifier le régime de rationalité qui se dégage de leur démarche. Nous y identifions plusieurs formes d'argumentation collaborative, et montrons que, bien que la rationalité dialogique et pratique propre aux discussions du texte talmudique lui-même fasse défaut dans le cadre de la chavruta, leur quête de vérités par le biais de discussions critiques autour des textes qui invitent à les découvrir révèle une rationalité ancrée dans des croyances religieuses, et dans une éthique existentielle.

\section{INDEX}

Mots-clés: apprentissage en collaboration, argumentation autour de textes réfutationnels, éducation juive, rationalité dialogique

Keywords: argumentation around refutational texts, collaborative learning, dialogic rationality, Jewish education

\section{AUTHOR}

\section{BARUCH SCHWARZ}

Université hébraïque de Jérusalem 